Open Access

\title{
Segmentation of epithelial human type 2 cell images for the indirect immune fluorescence based on modified quantum entropy
}

\author{
Abu-Zinadah Hanaa ${ }^{1,2^{*}}$ (D) and Abdel Azim Gamil ${ }^{3}$
}

\author{
*Correspondence: \\ habuzinadah@kau.edu.sa; \\ gazim3@gmail.com \\ ${ }^{1}$ Department of Statistics, Faculty of \\ Science- AL Faisaliah, King \\ Abdulaziz University, 32691, Jeddah, \\ Saudi Arabia \\ ${ }^{2}$ University of Jeddah, College of \\ Science,Department of Statistics, \\ Jeddah, Saudi Arabia \\ Full list of author information is \\ available at the end of the article
}

\begin{abstract}
The autoimmune disorders such as rheumatoid, arthritis, and scleroderma are connective tissue diseases (CTD). Autoimmune diseases are generally diagnosed using the antinuclear antibody (ANA) blood test. This test uses indirect immune fluorescence (IIf) image analysis to detect the presence of liquid substance antibodies at intervals the blood, which is responsible for CTDs. Typically human alveolar epithelial cells type 2 (HEp2) are utilized as the substrate for the microscope slides. The various fluorescence antibody patterns on HEp-2 cells permits the differential designation-diagnosis. The segmentation of HEp-2 cells of IIf images is therefore a crucial step in the ANA test. However, not only this task is extremely challenging, but physicians also often have a considerable number of IIf images to examine.

In this study, we propose a new methodology for HEp2 segmentation from IIf images by maximum modified quantum entropy. Besides, we have used a new criterion with a flexible representation of the quantum image(FRQI). The proposed methodology determines the optimum threshold based on the quantum entropy measure, by maximizing the measure of class separability for the obtained classes over all the gray levels. We tested the suggested algorithm over all images of the MIVIA HEp 2 image data set.

To objectively assess the proposed methodology, segmentation accuracy (SA), Jaccard similarity (JS), the F1-measure,the Matthews correlation coefficient(MCC), and the peak signal-to-noise ratio (PSNR) were used to evaluate performance. We have compared the proposed methodology with quantum entropy, Kapur and Otsu algorithms, respectively.The results show that the proposed algorithm is better than quantum entropy and Kapur methods. In addition, it overcomes the limitations of the Otsu method concerning the images which has positive skew histogram. This study can contribute to create a computer-aided decision (CAD) framework for the diagnosis of immune system diseases

(Continued on next page)
\end{abstract}

Springer Open

(c) The Author(s). 2021 Open Access This article is licensed under a Creative Commons Attribution 4.0 International License, which permits use, sharing, adaptation, distribution and reproduction in any medium or format, as long as you give appropriate credit to the original author(s) and the source, provide a link to the Creative Commons licence, and indicate if changes were made. The images or other third party material in this article are included in the article's Creative Commons licence, unless indicated otherwise in a credit line to the material. If material is not included in the article's Creative Commons licence and your intended use is not permitted by statutory regulation or exceeds the permitted use, you will need to obtain permission directly from the copyright holder. To view a copy of this licence, visit http://creativecommons.org/licenses/by/4.0/. 
(Continued from previous page)

Keywords: Quantum information, Image segmentation, Thresholding, Quantum entropy, Immune fluorescence images

\section{Introduction}

In recent years, much automation machinery was designed for all the most important steps in the IIf images analysis [1-8]. Classification models and techniques are used to recognize the mitotic cells, quantify the fluorescence intensity, and categorize the fluorescent patterns. Image segmentation is one of the first important and difficult steps during a computer-system diagnostic system using the IIf images. The approaches of cell HEp-2 segmentation is divided into 3 classes. The first class is the simplest procedure; it is supported by a thresholding result from morphological operations $[9,10]$. This method has restricted accuracy in the cells detection with non-uniform intensity patterns (e.g., light and dark areas in the same cell). The second class tries to overcome the limitation of the first class by splitting segmentation method into two or several steps (see $[3,5,8]$ ) or by utilizing the watershed algorithm [6]. In these approaches, there are several parameters dependent on the data sets, and they do not address the segmentation of complex models $[3,11]$. Finally, the third proposed approach is primarily based on the strategies of machine learning classification [7]. Generally, the techniques of Cell HEp-2 segmentation are evaluated on IIf images, which have complete distinct characteristics regarding the resolution, the contrast, and the level of noise and fluorescent patterns. In [7], the most significant segmentation techniques are assessed on IIf image database. The results of the assessments show that the segmentation accuracy of HEp-2 cells with completely distinct intensity levels and fluorescence patterns remain challenging. Several studies confirmed that diagnostic responsibility is severely affected by judgment, and also the variability of human analysis, thus requiring automatic identification techniques [12-14]. Quantum computing $(\mathrm{QC})$ is a novel field that combines information processing and physics. The difficult task is the secret writing data of quantum objects (called qubits). Nowadays, the quantum computer is far from complete. That being said, several existing classical computation algorithms can be improved by quantum computing, as it is. New techniques for quantum information processing are based on quantum machine learning, such as quantum state classification. It is one among quantum computing perspective [15]. The quantum information system (QIS) is a domain which becomes visible from previous the science of information and physics. Besides, QIS has transformed information science [16]. Existing algorithms for image processing can be improved by quantum mechanics of mathematical modeling [17]. The power of quantum computation can help to overcome many difficult tasks for classical computers. The information theory can consider as quantum computation applications [18]. Image processing has been studied extensively in classical computers [19]. Due to quantum parallelism, quantum computers could reduce the time complexity of linear, or even exponential algorithms that are run on classical computers [20-25]

In the literature, most research is concerning the image process operations, such as, representation, image retrieval [26], and storage and geometric transformations [27, 28]. 
In [29], the author proposed an algorithmic program based on the quantum signal process to find the image edge in a medical image. Also, Chenggang Yan et al. [30] proposes a method for estimating 2D layout and estimating 3D layout. The 2D layout estimation, is essentially a semantic edge detection based on the Pyramid Scene Parsing Network (PSPNet) [31]. The 2-layout estimation proposed as a regression problem of three types of edges. In [30], the authors confirmed that the results of their method are sharper than that of semantic transfer [32]. In [33, 34], Eldar and Oppenheim are first instructed quantum signal process systems. The entropy is a tool to measure the data information in a digital image from the perspective of quantum informatics. In [21] the author proposed an algorithm for image thresholding using quantum entropy $(\mathrm{QE})$. Additionally, the authors show that the advantages of quantum entropy than Shannon entropy. Also, QE is more accurate than Shannon entropy to measure image information.

The authors in [35] developed a new approach to image edge detection based on quantum entropy. When we tested the same methodology to segment the H2p-cells, we had unsatisfactory results which is clear in our simulations examples. Then, we developed another methodology based on quantum entropy modified with a new criteria (function) (equations 20 and 21). The new proposed methodology gave a good result compared to the other classical methods such as the Otsu and Kapur method. This study is structured as follows. In Section 2, digital images quantization (quantum entropy measures, optimal threshold selecting utilizing the proposed criterion, quantum state description for a gray scale image) was presented. Materials and methods (image segmentation algorithm base on modified quantum entropy, description of MIVIA HEp2 set, performance measures) are presented in Section 3. In Section 4, experimental results and discussion (numerical examples) the optimal are presented. Finally, Section 5 concludes with a summary of our work.

\section{Digital image quantization}

Quantum computing manipulates and analyses quantum objects (qubits) to solve computational problems. Quantum theory explains our observations at the quantum level when Newton's mechanics prove to be insufficient. Quantum computing utilizes the electrons, and photons which allows for more efficient of data handling on a tiny scale [18, 36]. Qubits are quantum objects, which have two distinct states and can also be in both states at the same time (principle of superposition).A bit in a classical computer is analogous to a qubit in a quantum system. In the notation of the quantum states, the general noiseless qubits look like:

$$
|\psi\rangle=\alpha|0\rangle+\beta|1\rangle
$$

where $\alpha$ and $\beta$, are arbitrary complex numbers with $|\alpha|^{2}+|\beta|^{2}=1 \alpha, \beta$ are probability amplitudes and $\psi$ is arbitrary superposition (linear combinations) of the twos states $|0\rangle$ and $|1\rangle$. Using the Dirac notation, $|0\rangle$ and $|1\rangle$ states are called kets. There are a number of quantum image representations that a quantum computer may use. Similar to pixel representations on traditional computers, quantum computers use flexible representation of quantum images (FRQI), multi-channel representation for quantum image (MCQI), and novel enhanced quantum representation of digital images (NEQR) to convert an image into quantum representation [36-41]. In [21], the author presents a novel technique that uses maximum quantum entropy to detect of suspicious regions in digital mammograms; 
in [35], the authors developed a new approach to image edge detection based on quantum entropy. In this paper, we used the FRQI to represent an image. We keep that line of research and developed a modified of quantum entropy to segment the HEp2 cell images. This technique helps store classical images in quantum computer representations that recognize the colors and the corresponding positions of the image's pixels. A flexible representation of a quantum image (FRQI) has proposed by the authors [36]. This is an essential step to being able to use quantum techniques on any classical image. FRQI is define as:

$$
\begin{aligned}
|\psi\rangle & =\frac{1}{2^{n}} \sum_{i=0}^{2^{2 n}-1}\left|c_{i}\right\rangle \otimes|i\rangle \\
& =\frac{1}{2^{n}} \sum_{i=0}^{2^{2 n}-1}\left(\cos \theta_{i}|0\rangle+\sin \theta_{i}|1\rangle\right) \otimes|i\rangle
\end{aligned}
$$

In this process, the qubits in the quantum image correspond to the pixels by:

$$
\||\psi\rangle \|=\frac{1}{2^{n}} \sqrt{\sum_{i=0}^{2^{2 n}-1}\left(\cos ^{2} \theta_{i}+\sin ^{2} \theta_{i}\right)}=1,
$$

where $\theta=\left(\theta_{1}, \theta_{2}, \ldots, \theta_{2^{2 n}}-1\right)$ and $\theta_{i} \in[0, \pi / 2]$ is the angle vector that encodes the color information. The color of each pixel in an image is:

$$
\left|c_{i}\right\rangle=\left(\cos \theta_{i}|0\rangle+\sin \theta_{i}|1\rangle\right)
$$

and the states

$$
|i\rangle=|0\rangle,|1\rangle,|2\rangle, \downarrow, \cdot,\left|2^{2 n}-1\right\rangle
$$

represent the position in a sequences of base state of number of qubits. Now, we show how to use the FRQI model in a normalized wave function $|\psi\rangle$ and obtain the density matrix $\rho$, from which we are able to calculate the von Neumann entropy. According to this model, the wave function is composed of the qubit of color $q_{c}$ characterized by the parameter $\theta \in[0, \pi / 2]$ and the mesh of qubits of position $\mid i>$. Using a transformation $[0,255]$ to the domain $[0, \pi / 2]$, we have $\theta_{i}=(\text { color })_{i} \times \frac{\pi}{2} \times \frac{1}{255}$ and $q_{c}=\left|c_{i}\right\rangle=\sin \left(\theta_{i}\right)|1\rangle+\cos \left(\theta_{i}\right)|0\rangle$. In this process, a qubit in the quantum image corresponds to a pixel by:

$$
\left.\left|(\psi\rangle=\cos \left(\theta_{m}\right)\right| 0\right\rangle+\sin \left(\theta_{m}\right)|1\rangle
$$

where $\theta_{m} \in\left[0, \frac{\pi}{2}\right], m=o, 1, \ldots, 2 n-1$. The Bloch sphere representation of a qubits. Suppose $f(i, j)$ are normalize pixels values of the original gray scale image. $f(i, j) \in\left[0, \frac{\pi}{2}\right]$, where $(i, j)$ are pixels coordinates, with $\theta_{m}=\frac{\pi}{2} f(i, j)$ equation 6 can be rewritten as:

$$
\left.\left.\left|(\psi\rangle=\cos \left(\frac{\pi}{2} f(i, j)\right)\right| 0\right\rangle+\sin \left(\frac{\pi}{2} f(i, j)\right)\right)+|1\rangle
$$

We can represent any gray scale image as a two-level quantum system using Eq. 7. This allows us to use any quantum algorithms on the image.

\subsection{Quantum entropy measures}

Quantum entropy measures (QEM) estimate the the uncertainty of a quantum state. Shannon entropy used to measure the amount of information for a variable. Given a 
quantum system, $\Psi>$ that has $\mathrm{N}$ states $\left|\psi_{1}\right\rangle,\left|\psi_{2}\right\rangle, \ldots\left|\psi_{N}\right\rangle$ where the $N$ states have probabilities $P_{1}, P_{2}, \ldots, P_{N}$, quantum information system can be communicated as:

$$
\left|\psi_{1}\right\rangle,\left|\psi_{2}\right\rangle, \ldots\left|\psi_{N}\right\rangle \quad \text { with } P_{1}, P_{2}, \ldots, P_{N}
$$

The density operator of a quantum information system is given by:

$$
\rho=\sum_{i=1}^{N} P_{i}\left|\psi_{i}\right\rangle\left\langle\psi_{i}\right|
$$

Quantum entropy for a quantum information system $\Psi\rangle$ is given by $[42,43]$ :

$$
S(\rho)=-\operatorname{tr}(\rho \log (\rho))
$$

where $\mid>$ is the ket of Dirac notations and $\rho$ indicates the density matrix of the quantum $|\Psi\rangle$ system. With $0 \log 0=0$ when $\rho=0$, quantum entropy (QE) can measure the non-neighborhood relationship between quantum frameworks or quantum bases. The Shannon entropy is not as accurate as quantum entropy (QE), where Shannon entropy is defined by:

$$
H(X)=H\left(p_{1}, p_{2}, \ldots, p_{N}\right)=\sum_{j=1}^{N} p_{j} \log \left(p_{j}\right)
$$

where $p_{1}, p_{2}, \ldots, p_{N}$ are probabilities of the variable $X$. We consider the connections between the pixels in digital images as the cross-correlations of qubits in quantum systems. The quantum entropy of the digital image can take relationship properties among pixels into the calculation whereas Shannon entropy is powerless [35, 44]. The quasithreshold that leads to maximum quantum entropy converges to the perfect threshold, where the maximum sum of information obtained under these conditions.

\subsection{Optimal threshold selecting utilizing the proposed criterion}

Accept that the 8-bit profundity interesting gray-scale image comprises of pixels. The pixels found at $(i, j)$ positions with its gray scale value. Probabilities of each gray scale are calculated as:

$$
p_{i}=\frac{\sum_{i=1}^{L} f_{i}}{M N}
$$

where $L$,indicate numbers of the pixels and $f_{i}$ are the gray scale values of pixel $\mathrm{i}$. At that point, 256 angles are characterized as taking after to encode 256 gray scales.

$$
\theta_{m}=\frac{\pi}{2} p_{m}=\frac{\pi \sum_{j=1}^{m} f_{j}}{2 N M}
$$

$\mathrm{QE}$ of the $j$ th quasi-segment is gotten by $S(\rho)=-\operatorname{tr}(\rho \log (\rho))$ and the esteem of QE of quasi-segments is:

$$
S(\rho)=\sum_{m=1}^{j-1} S\left(\rho_{m}\right)
$$




\subsection{Quantum state description for a gray scale image}

Let us take $n$ is the quasi-segments and the quasi-thresholds are signified by $t_{1}, t_{2}, \ldots, t_{n-1}$ . In this case the quantum state of the $m$ th quasi-segment is

$$
\left.\left|I_{m}(\theta)\right\rangle=\sum_{j=0}^{m-1} \cos \left(\theta_{j}\right)|\rangle+\left|\sin \left(\theta_{j}\right)\right|\right\rangle
$$

where $m=1,2, \ldots, 2^{n}-1$, and density operator of the $m$ th quantum state is calculated as:

$$
\rho_{m}=P_{m}\left|I_{m}(\theta)\right\rangle\left\langle I_{m}(\theta)\right|
$$

where $P_{m}=\sum_{i=1}^{m} p_{i}$, and $p_{i}$ calculate by Eq. 12 . QE of the $m$ th quasi-segment, specifically, $S\left(\rho_{m}\right)$ is accomplished by Eq. 13, and gross value of QE of these m quasi-segments is accomplished as:

$$
S(\rho)=\sum_{j=1}^{m} S\left(\rho_{j}\right)
$$

Then values of final thresholds $t_{1}, t_{2}, \ldots, t_{n}$ are obtained from quasi-thresholds which lead to the maximum esteem of $S(\rho)$, namely, values of final thresholds $T_{j}=t_{j}$ if and only if the condition of $S(\rho)=\max (S \rho)$ is satisfied. Let IM denote a gray scale image with $M$ gray levels $[0,1, \ldots, M-1]$. The number of pixels with gray level i denoted by $n_{i}$ and $N$ equal to the total number of pixels $\left(N=n_{0}+n_{1}+\ldots+n_{M}\right)$. The probability of gray level $\mathrm{j}$ showed up within the image is defined as: $p_{j}=\frac{n_{j}}{N}, p_{j}>0, \sum_{j=0}^{N} p_{j}=1$ assume the pixels of the image are separated into two classes $\mathrm{O}$ and $\mathrm{B}$ by a gray level s. $\mathrm{O}$ is the pixels with levels $\{0,1, \ldots, s\}$, and the rest of the pixels belongs to $B$, where $O$ and $B$ are

typically corresponding to the object class and the background one $P_{O}=\frac{p_{1}}{W_{1}}, \frac{p_{2}}{W_{2}}, \ldots \ldots \frac{p_{s}}{W_{s}}$ and $P_{B}=\frac{p_{s+1}}{W_{s+1}}, \frac{p_{s+2}}{W_{s+2}}, . ., \frac{p_{M-1}}{W_{M-1}}$ where $W_{1}(s)=\sum_{i=0}^{s} p_{i}$ and $W_{2}(s)=1-W_{1}(s)$ and $s$ is the threshold value. According to the definition of QEM, the density operator of background pixels and the object region pixels can characterize as follows:

$$
\rho_{j}=\left|I_{j}(\theta)\right\rangle P_{j}\left\langle I_{j}(\theta)\right|, \text { where } j=O, B
$$

And the quantum entropy measure (QEM) of background pixels and the object region pixels are

$$
S(\rho)=S\left(\rho_{O}\right)+S\left(\rho_{B}\right)
$$

The QEM $S(\rho)$ is parametrically dependent on the threshold value $\mathrm{t}$ for the object and background. When $S(\rho)$ is maximized, the luminance level $t$ is considered as the optimum threshold value. In this work, we have proposed a new criterion for QEM as the following:

$$
\begin{aligned}
S(\rho) & \left.=S\left(\rho_{O}\right)+S\left(\rho_{B}\right)+(1-q)^{\alpha} \max \left[0, S\left(\rho_{O}\right) S\left(\rho_{B}\right)\right], q \in\right] 0,1[ \\
S(\mathrm{opt}) & =\operatorname{argmax}[S(\rho)]
\end{aligned}
$$

and the maximum value of $S(\rho)$ is the image thresholding.

\section{Materials and methods}

In the following, the algorithm as well as the data set used for segmentation are described. 
3.1 Image segmentation algorithm base on modified quantum entropy

In this section, we define the proposed algorithm as flowing:

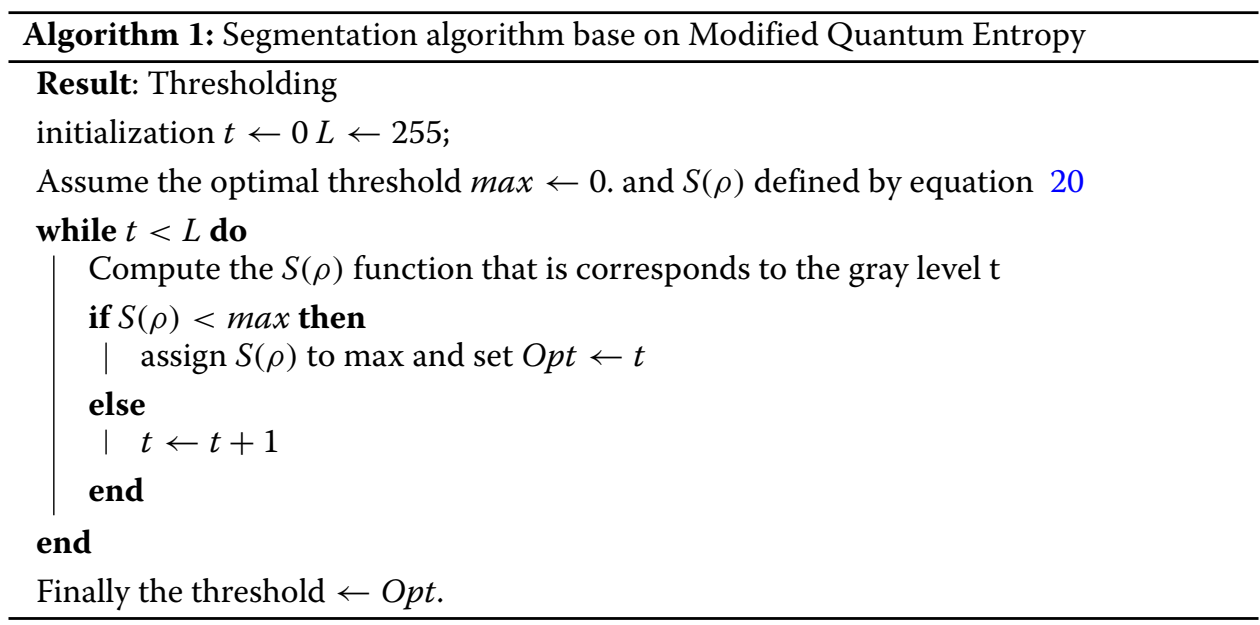

\subsection{Description of MIVIA HEp2 image data set}

We have utilized MIVIA HEp2 image data set consists of 28 images, where the images have a resolution of $1388 \times 1038$ pixels, a color depth of 24 bits and are stored in stored as bitmap images. The images were obtained by means of a fluorescence microscope (40-fold amplification) coupled with a 50W mercury vapor lamp and with a digital camera. The camera contains a CCD with a squared pixel of equal side to $6.45 \mu \mathrm{m}$. The six type of HEp2 (centromere, nucleolar, homogeneous, fine speckled, coarse speckled, and cytoplasmic) presented and tested [45].

\subsection{Performance measures}

After reading and reviewing some references related to segmentation evaluation criteria. We can divide it into three groups as following: (1) Subjective methods (observation methods). It depends on personal vision. (2) Supervised methods (statistical standards) need a gold standard image (personal expert) to compare with the segmentation result. This group overcomes the limitations of the observation methods, So it has been used in many papers. The examples of this group are dice similarity coefficient (DSC), Jaccard similarity (JS), specificity (SP), sensitivity (SE), F1-measure, and segmentation accuracy (SA), and they are the most commonly used ones. (3) Unsupervised methods based on the measure of the similarity between the segmented image and original image, for example the peak signal-to-noise ratio (PSNR). In this paper, we have adopted all three types to assess the proposed method as the following-observation method, segmentation accuracy (SA), Jaccard similarity(JS), F1-measure, Matthews correlation coefficient (MCC), and the peak signal-to-noise ratio (PSNR) [46].

$$
\begin{aligned}
\operatorname{Accuracy}(\mathrm{SA}) & =\frac{T P+T N}{F N+F P+T P+T N} \\
\text { Dice similarity coefficient }(\mathrm{DSC} / \mathrm{F} 1) & =\frac{2 * T P}{2 * T P+F P+F N}
\end{aligned}
$$

it is the measure of degree for overlapping between two images, if $D S c=1$ perfect match, $D S C=0$ no match. 


$$
\operatorname{Jaccard}(J S)=\frac{\text { Dice }}{2-\text { Dice }}
$$

it is used to compare similarity and diversity of image, given by the intersection to the union, JS generally comes between 0 and 1 , so, a higher JS is for better segmentation.

$$
\begin{aligned}
\text { Precision } & =\frac{T P}{T P+F P} \\
\text { Sensitivity - Recall - True positive rate (TPR) } & =\frac{T P}{T P+F N}
\end{aligned}
$$

where, for a given class A (foreground or background),

True positive (TP) : pixels correctly segmented as A.

False positive (FP): pixels falsely segmented as A.

True negative (TN) : pixels correctly detected as not A.

False Negative (FN) : pixel classified incorrectly as not A.

Finally, the peak signal-to-noise ratio (PSNR): [11, 20].

$$
\text { PSNR }=20 \log \frac{255}{\text { RMSE }}
$$

where the RMSE is the root mean-squared error that is defined by: RMSE = $\sqrt{\frac{\sum_{i=1}^{M} \sum_{j=1}^{N}|S(i, j)-I M(i, j)|^{2}}{M N}}$ where S and IM are the segmented and the input images, respectively. A higher PSNR value indicates a better threshold image quality. We chose

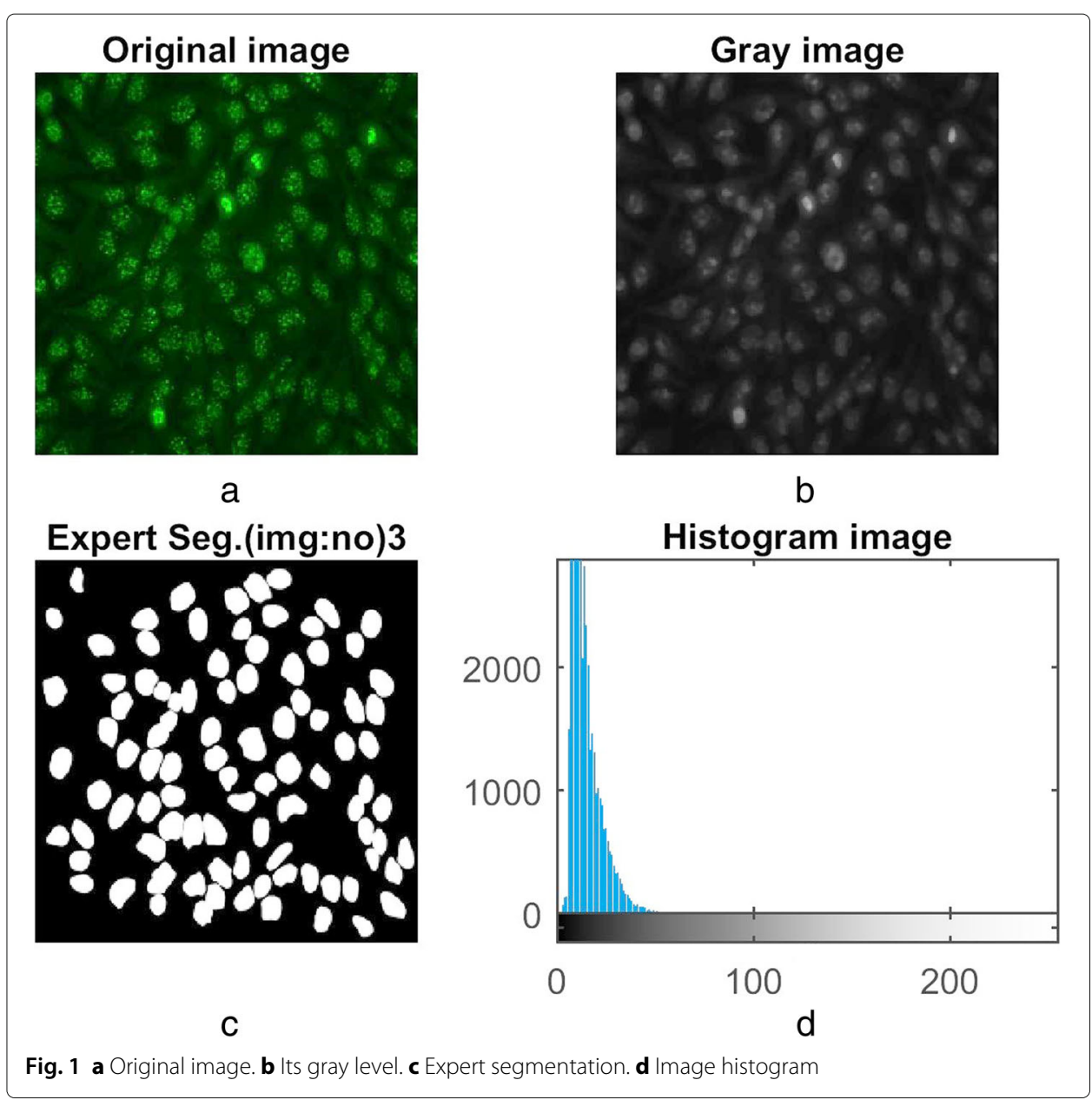




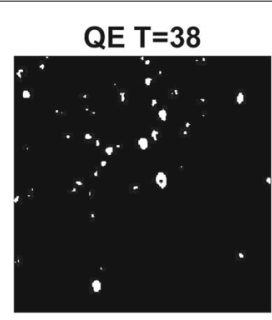

E

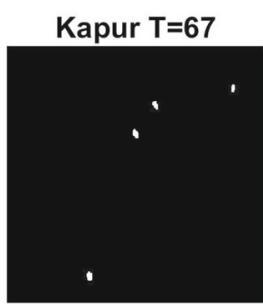

$\mathrm{H}$

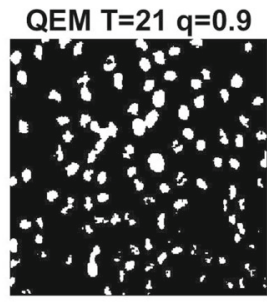

$\mathrm{F}$

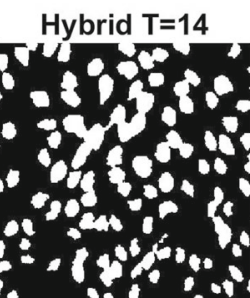

L

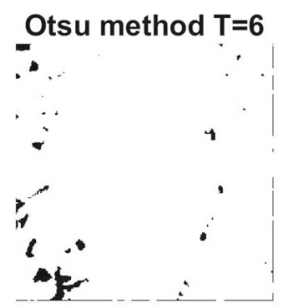

G

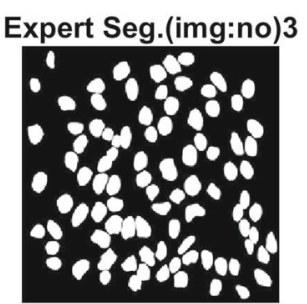

M

Fig. 2 F-measure [0.0865 0.53710 .42750 .01600 .7707$]$ for quantum entropy, modified quantum entropy (proposed), Otsu, Kapur, and hybrid of the proposed with Otsu methods, respectively

F-measure over precision and recall since it is the harmonic mean of precision and recall (TPR/sensitivity). The JS is very similar to dice as they are positively correlated, where Jaccard $=$ dice $/ 2$-dice. One disadvantage of the F-measure, a function of precision and recall, is that it is only interested in the positive class. All three measures (F-measure, recall and precision) use only 3 values (TP, FP, FN) without the 4th (TN).

$$
\mathrm{MCC}=\frac{T P * T N-F P * F N}{\sqrt{(T P+F P) *(T P+F N) *(T N+F P) *(T N+F N)}}
$$

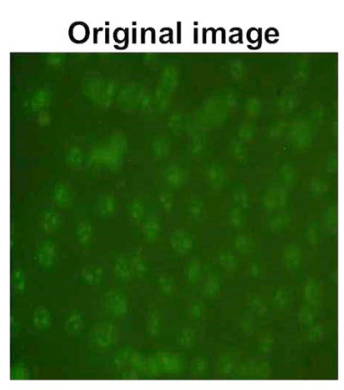

a

Expert Seg.(img:no)4

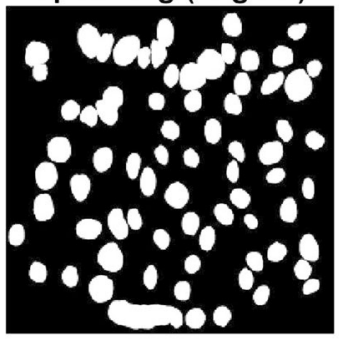

C

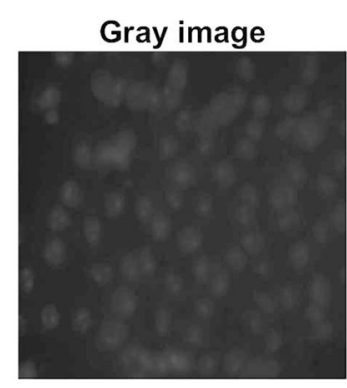

b

Histogram image

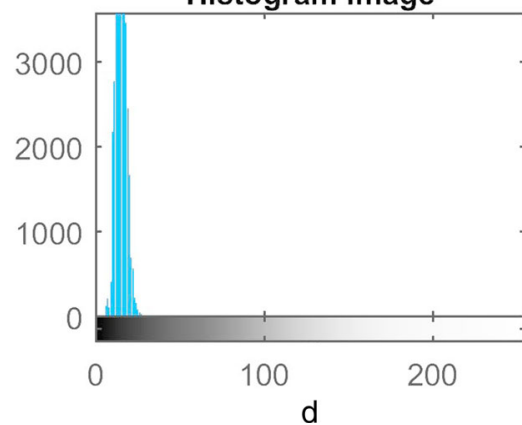

Fig. 3 a Original image. b Its gray level. c Expert segmentation. d Image histogram 


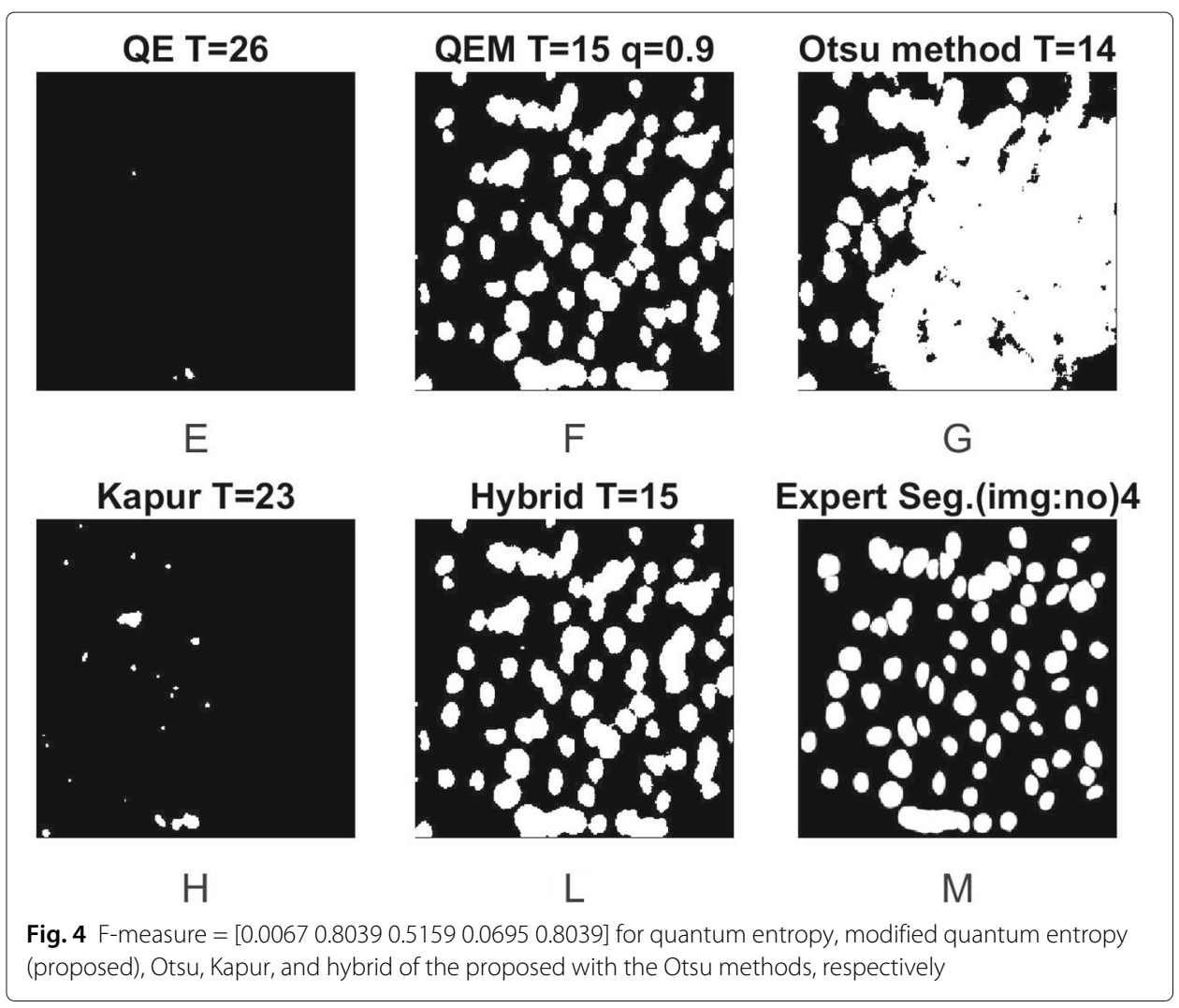

The Matthews correlation coefficient treat the true class (expert image) and segmented image class as two binary variables and find their correlation coefficient. The higher the correlation between true and segmented image, the better the segmentation. MCC considers all four values TP, TN, FP, and FN of confusion matrix and is belonging to $[-1,1]$. The closer the MCC value is to 1 , the better is the classification of both classes (object and background).

\section{Experimental results and discussion}

In the following, the numerical examples as well as the figures are described.

\subsection{Numerical examples}

For all examples, each example have two figures. For the first figure, we used the following notation: (a) original image, (b) its gray level, (c) expert segmentation, and (d) image histogram, while for the second figure, (e) quantum entropy, (f) quantum entropy modified (proposed), (g) Otsu method, (l) hybrid method, (h) Kapur method, and (m) segmentation by experts, where the hybrid method threshold is the average between the quantum entropy modified threshold and the Otsu threshold. In the segmentation by expert image, we write the number of the image in the data set. The values of F1measure of quantum entropy, quantum entropy modified (proposed),Otsu, Kapur, and hybrid methods, respectively, are written under the figures. In addition, the value of $q$ in (20) is shown in each figure with the value of threshold T. All the images of data set are tested. 


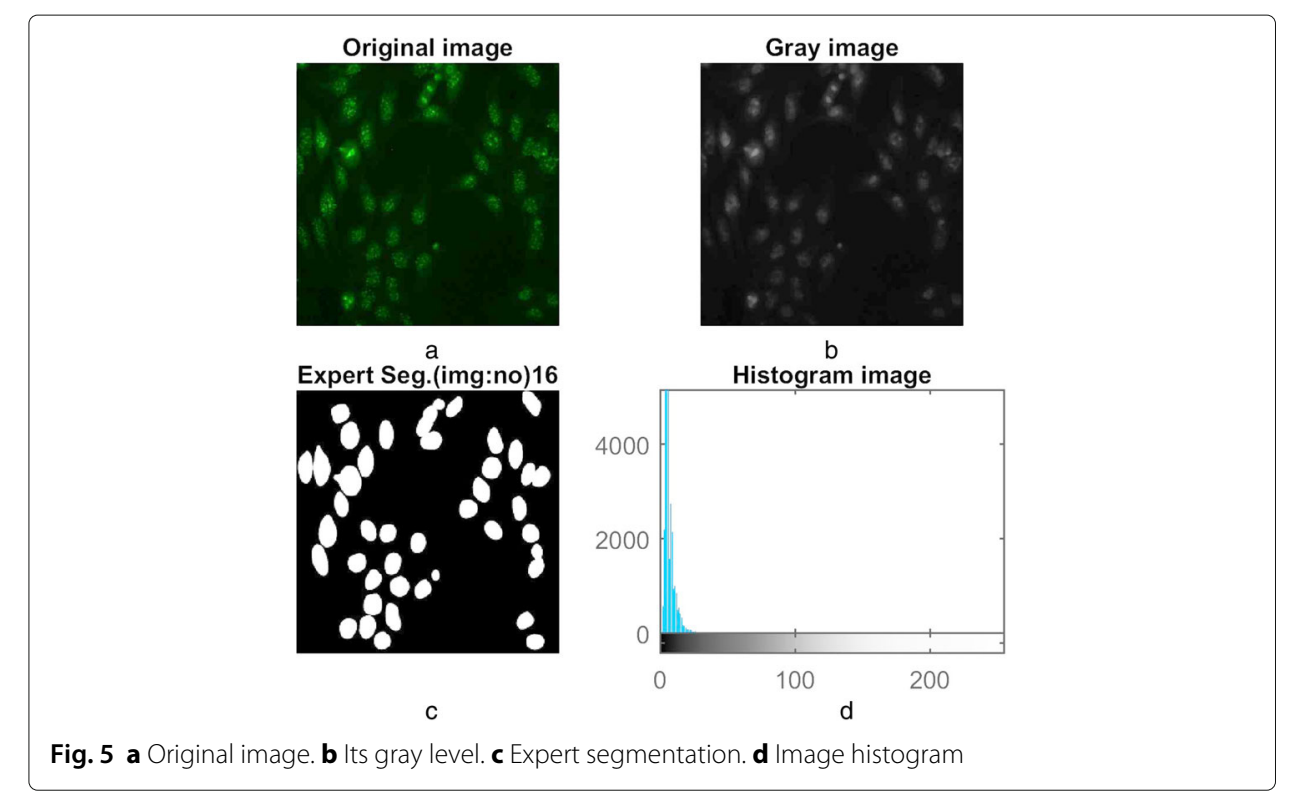

\subsubsection{Example 1}

(image no3): Figures 1 and 2. Histogram shape: Positive skew, QEM (proposed method) is better than QE, Kapur, and Otsu.

\subsubsection{Example 2}

(image no 4 ): Figures 3 and 4. Histogram shape: Bi modal, QEM (proposed method) is better than QE, Kapur, and Otsu.

\subsubsection{Example 3}

(image no 16 ): Figures 5 and 6. Histogram shape: Positive skew, QEM (proposed method) is better than QE, Kapur, and Otsu.

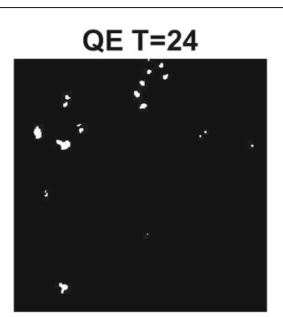

E

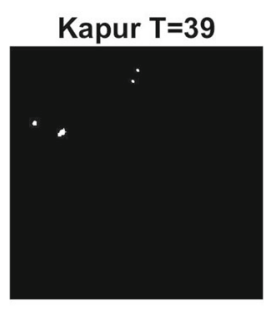

$\mathrm{H}$

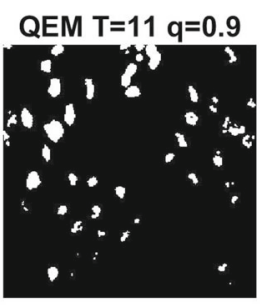

F

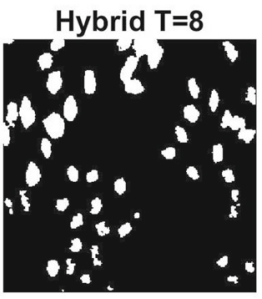

L

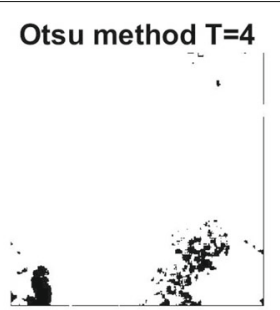

$\mathrm{G}$

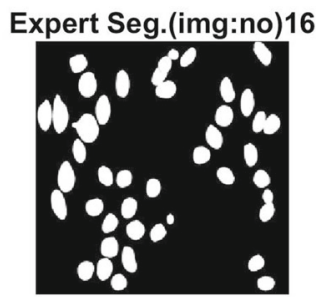

M

Fig. 6 F-measure $=\left[\begin{array}{l}0.06350 .44410 .31090 .01170 .6981\end{array}\right]$ for quantum entropy, modified quantum entropy (proposed), Otsu, Kapur, and hybrid of the proposed with the Otsu methods, respectively 


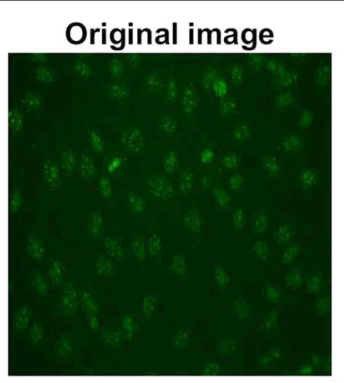

a

Expert Seg.(img:no)19

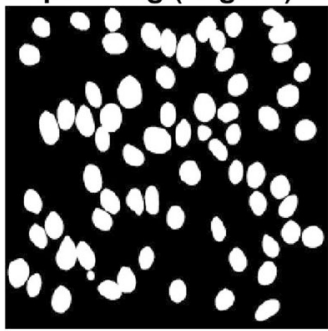

C

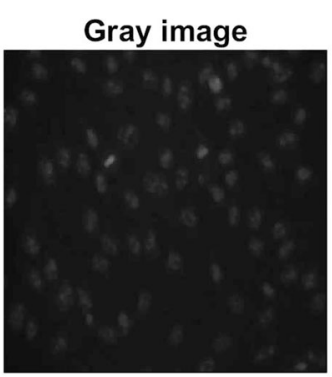

b

Histogram image

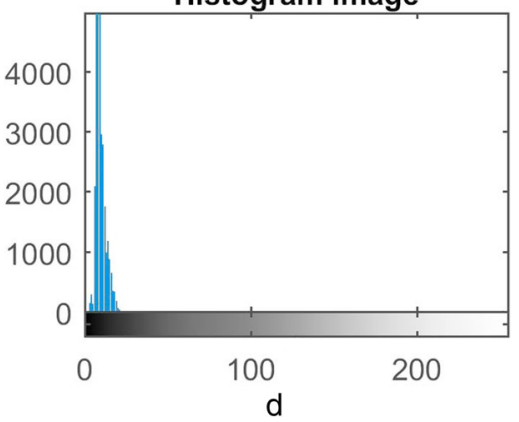

Fig. 7 a Original image. b Its gray level. c Expert segmentation. d Image histogram

\subsubsection{Example 4}

(image no 19 ): Figures 7 and 8. Histogram shape: Bi modal, QEM (proposed method) is better than QE, Kapur, and Otsu, and it is very near to expert segmentation.

\subsubsection{Example 5}

(image no 21): Figures 9 and 10 Histogram shape: Positive skew with double peaked, QEM (proposed method) is better than QE, Kapur, and Otsu.

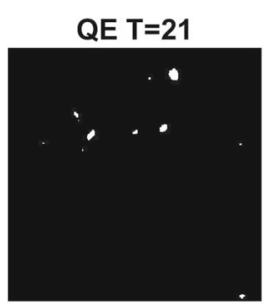

E

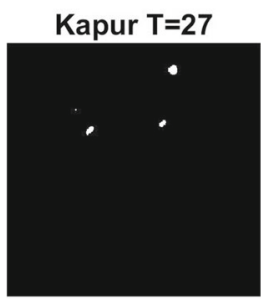

$\mathrm{H}$

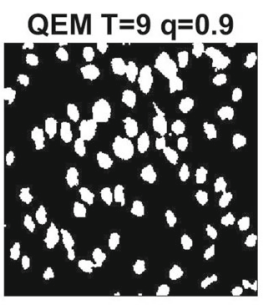

F

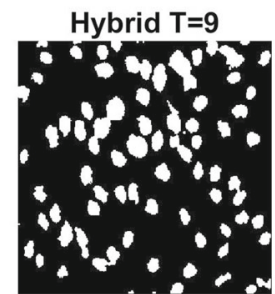

L

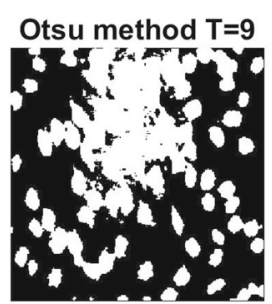

G

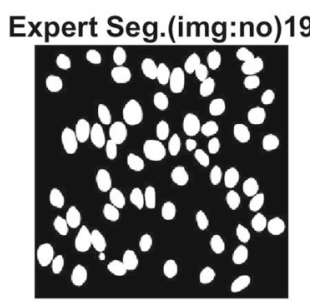

M

Fig. 8 F-measure $=[0.02870 .79880 .71780 .018690 .7988]$ for quantum entropy, modified quantum entropy (proposed), Otsu, Kapur, and Hybrid of the proposed with the Otsu methods, respectively 


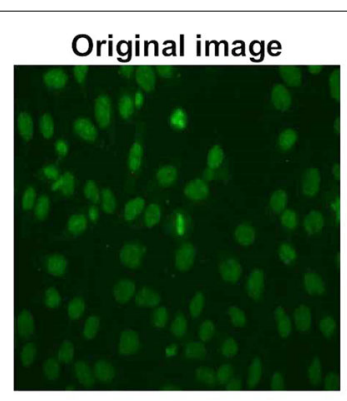

a

Expert Seg.(img:no)21

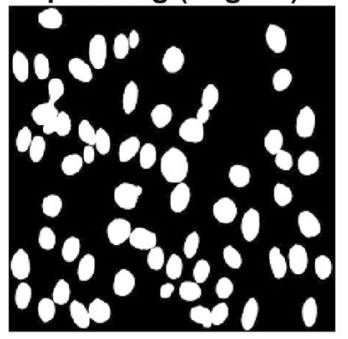

C

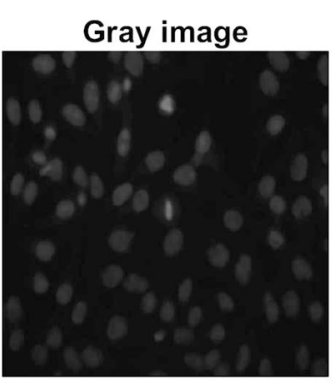

b

Histogram image

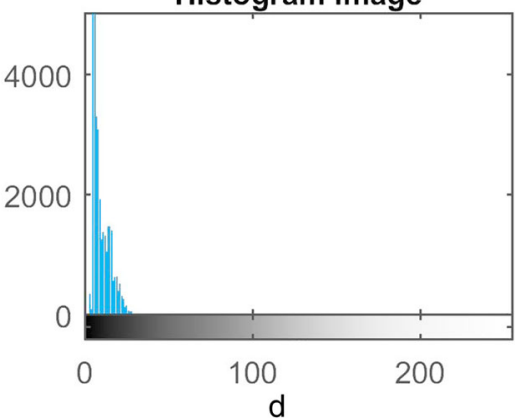

Fig. 9 a Original image. b Its gray level. c Expert segmentation. $\mathbf{d}$ Image histogram

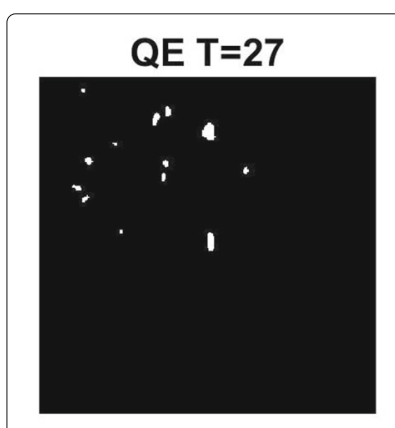

E

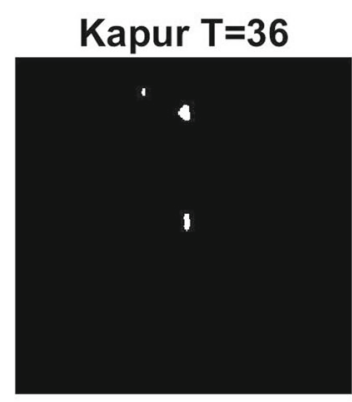

$\mathrm{H}$

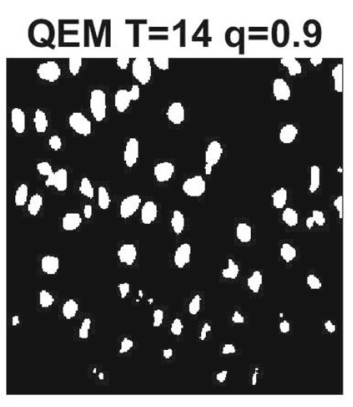

$\mathrm{F}$
Otsu method $\mathrm{T}=3$

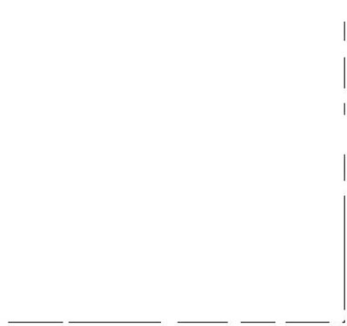

G

Expert Seg.(img:no)21

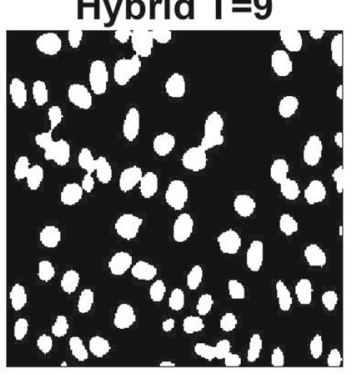

L

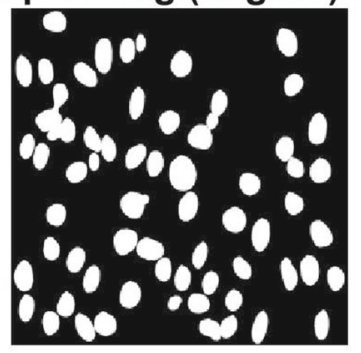

$\mathrm{M}$

Fig. 10 F-measure $=\left[\begin{array}{l}0.04850 .59410 .35180 .01940 .8438\end{array}\right]$ for quantum entropy, modified quantum entropy (proposed), Otsu, Kapur, and hybrid of the proposed with Otsu methods respectively 


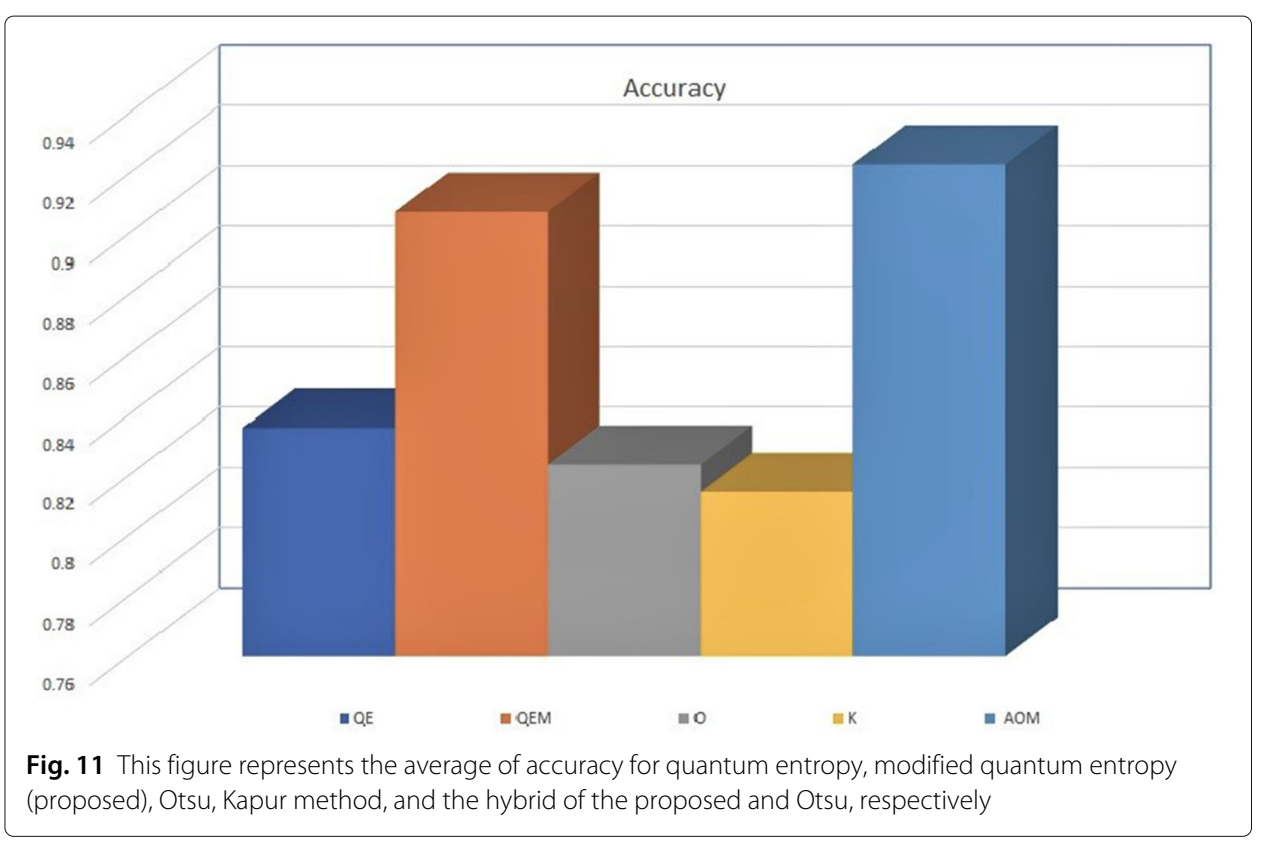

Based on the subjective (observation), supervised and unsupervised evaluation methods. In all examples and figures we observed that (1) the proposed method quantum entropy modified (QEM) is best than QE and Kapur over all data set. In addition, it is best than Otsu over $40 \%$ of data set. (2) Our observation is that the Otsu method has a problem to find a good thresholding for the image which has positive skew histogram. On the contrary, the QEM finds the best thresholding for the same images. (3) The quantum entropy modified is better than Otsu, for certain class of images which has positive skew histogram. See Figs. 1, 2, 3, 4, 5, 6, 7, 8, 9, and 10.

\subsection{Summarize table}

In the following, one table summarize all measurement PNSR, accuracy, F-measure, Jaccard, and Mcc.

\subsection{Summarize figures}

In the following, four figures to summarize the average of accuracy, F-measure, Jaccard, MCC, and PNSR measures over all data set images, which are correspond to the quantum entropy, modified quantum entropy (proposed), Kapur Otsu, and hybrid methods (Figs. 11, 12, 13, 14, and 15)

\subsubsection{Accuracy}

The following Fig. 11, represent the accuracy average of all images in the data set with all methods.

\subsubsection{F1-measure}

The following Fig. 12, represent F1-measure of all images in the data set with all methods.

\subsubsection{Jaccard}

The following Fig. 13, represent Jaccard measure of all images in the data set with all methods. 


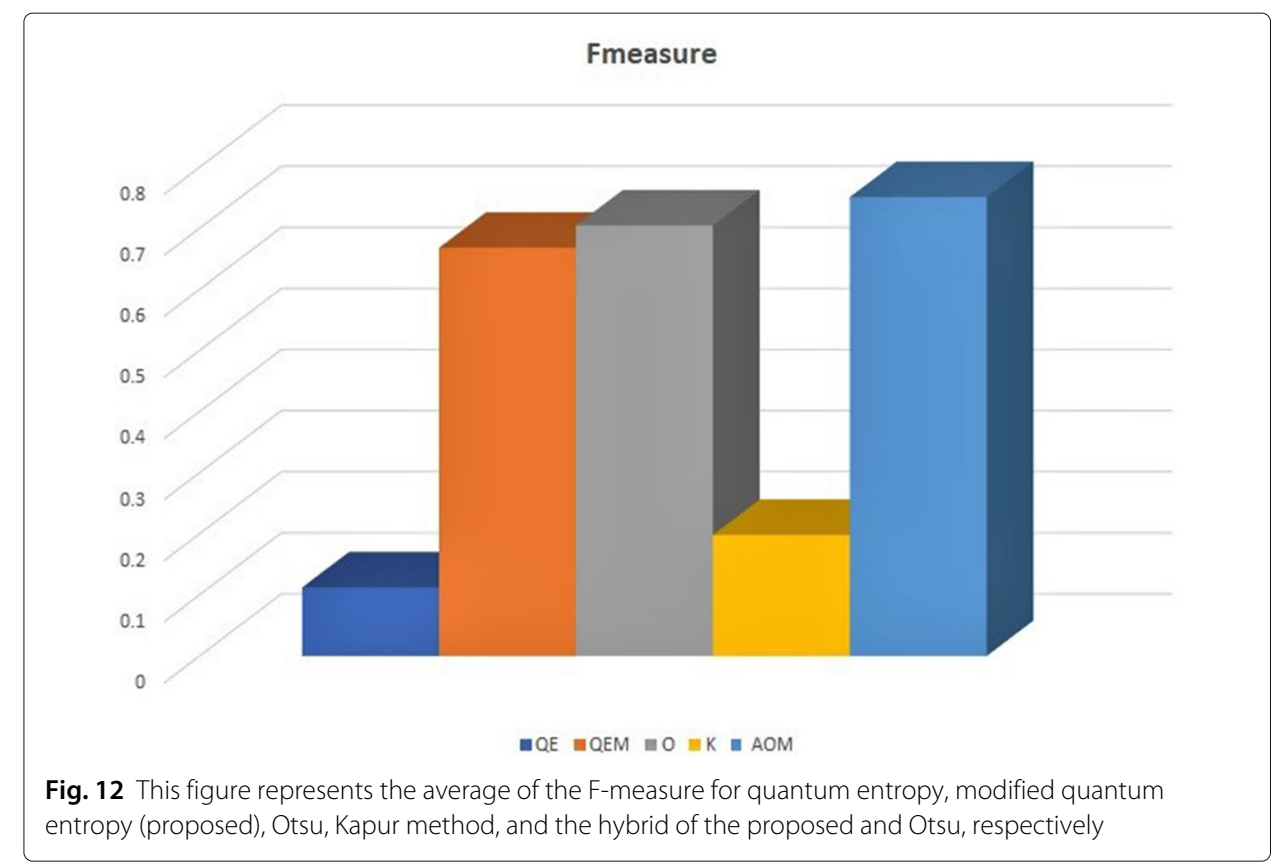

\subsubsection{MCC}

The following Fig. 14, represent average of the Matthews correlation coefficient (MCC) of all images in the data set with all methods.

\subsubsection{PNSR}

The following Fig. 15, represent PNSR measure of all images in the data set with all methods.

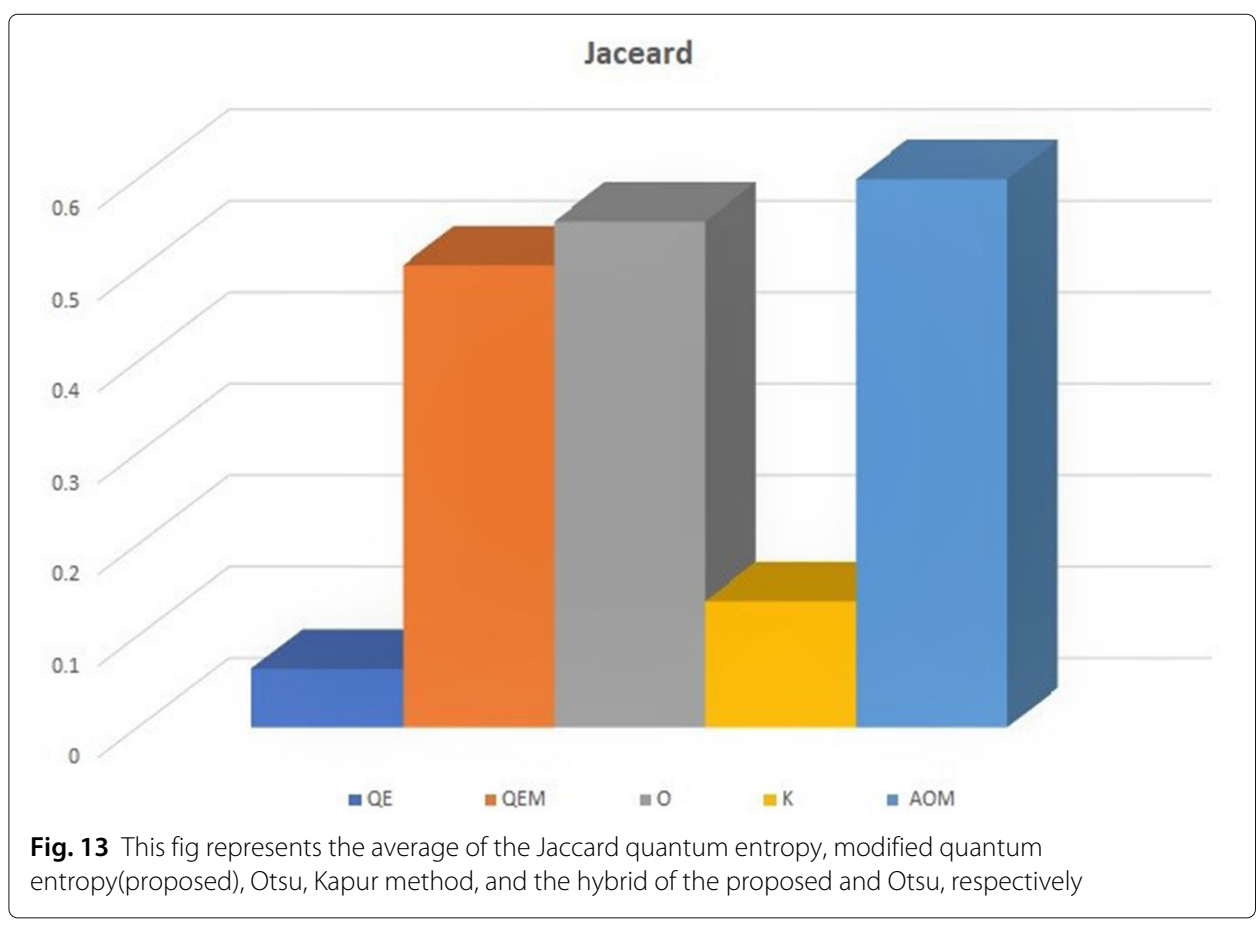




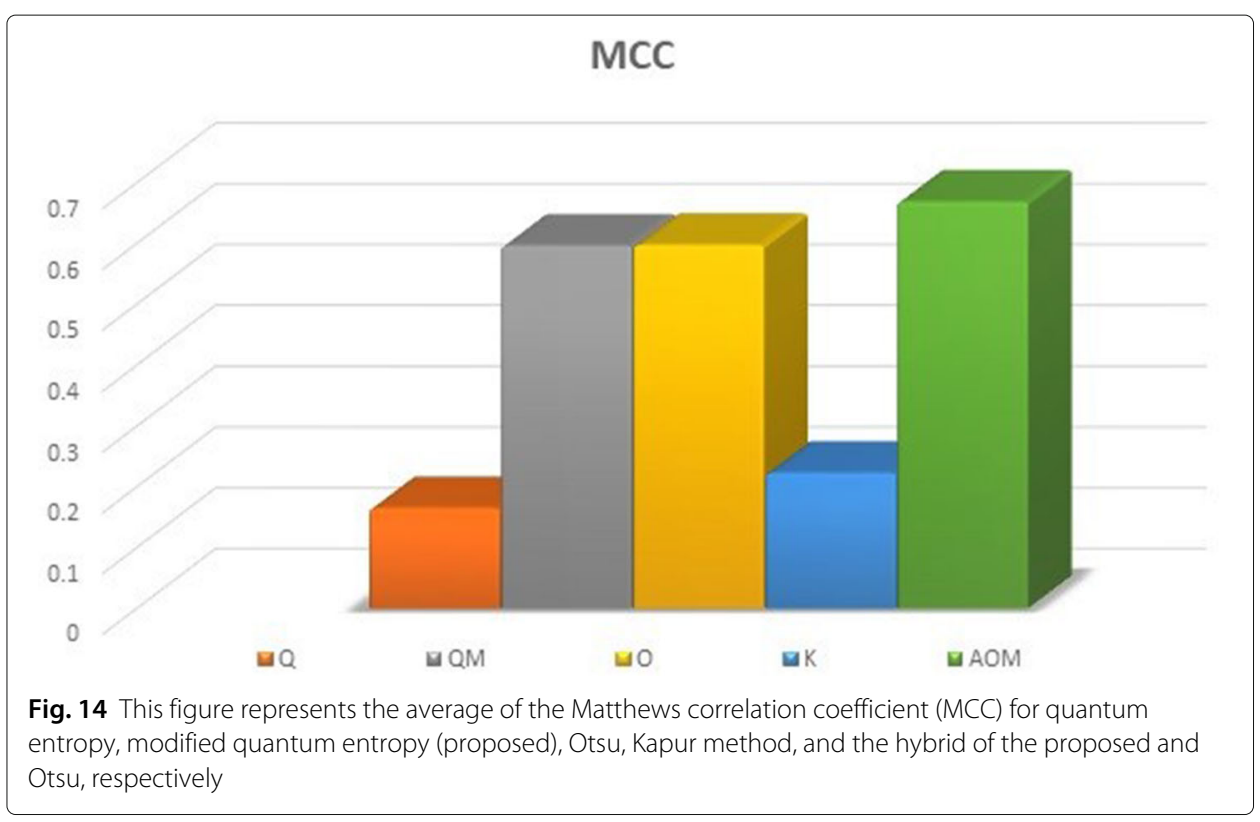

The experiments and Figs. 11, 12, 13, 14, and 15 of the average of accuracy, F1, Jaccard, MCC, and PNSR show that the QEM method is better than quantum entropy, and Kapur methods. See Table 1; it is very comparable to the Otsu method.

\section{Conclusion}

We developed an efficient methodology for HEp2 cell segmentation from IIf images based on maximum of new criterion quantum entropy modified and an adaptable representation of the quantum image (FRQI). The method was tested on all the images of the MIVIA HEp 2 image data set. The results showed that the proposed method is most accurate

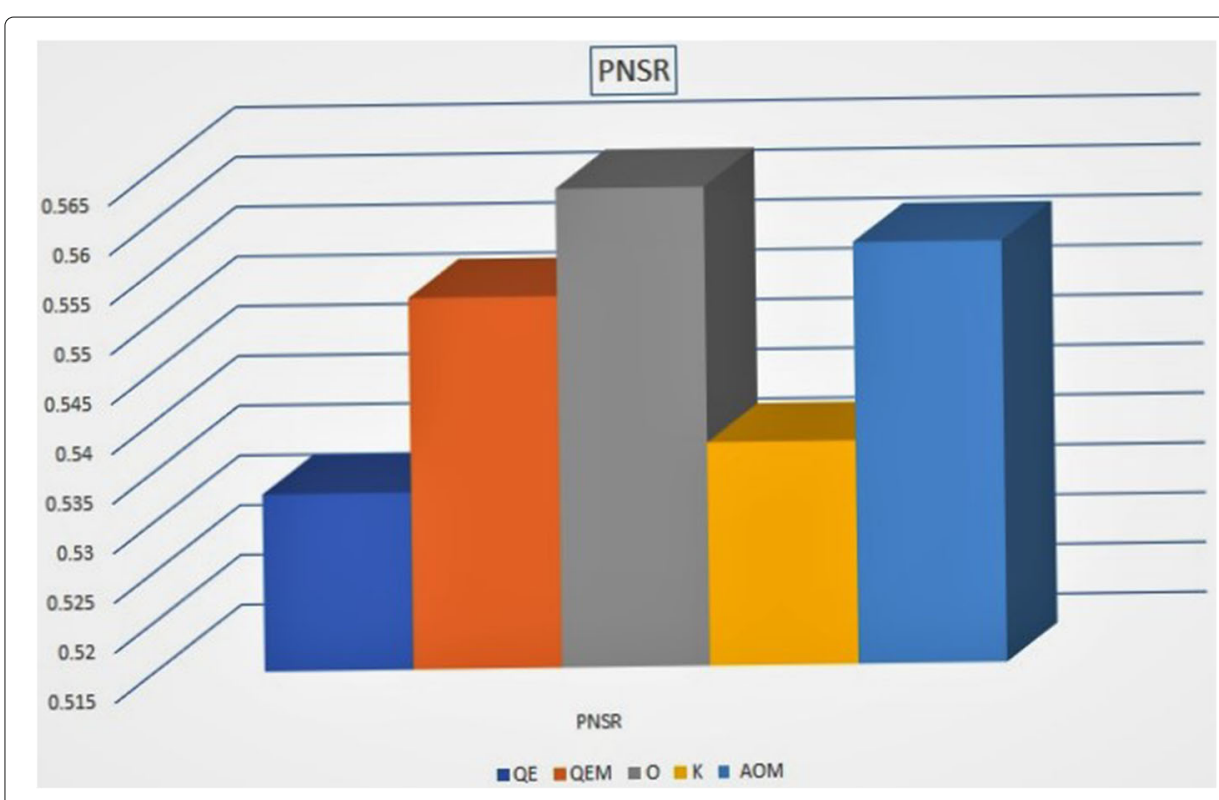

Fig. 15 This figure represents only the average of decimal part of the PSNR for quantum entropy, modified quantum entropy(proposed), Otsu, Kapur method, and the hybrid of the proposed and Otsu, respectively 
Table 1 average of PSNR, accuracy, F-measure, Jaccard and MCC for quantum entropy, quantum modified entropy(proposed), Otsu, Kapur method, and the hybrid methods respectively

\begin{tabular}{llllll}
\hline Avg & QE & QEM & O & K & AOM \\
\hline PNSR & 54.19561 & 54.20799 & 54.22972 & 54.19427 & 54.22237 \\
Accuracy & 0.746429 & 0.821304 & 0.292297 & 0.737946 & 0.874832 \\
F-measure & 0.086522 & 0.537096 & 0.427464 & 0.016042 & 0.770693 \\
Jaccard & 0.045217 & 0.367144 & 0.271831 & 0.008086 & 0.62693 \\
Mcc & 0.016 & 0.596 & 0.60 & 0.22 & 0.67 \\
\hline
\end{tabular}

of quantum entropy and Kapur methods in all examples. The proposed method overcomes the limitations of Otsu method Concerning the images which has positive skew histogram. The QEM finds the best thresholding than Otsu for the images which has positive skew histogram. Also, the experimental results show that the proposed methodology entropy outperforms the other strategies and gives superior segmentation output.The study can be improved by a new function with good parameters. This study can be utilized in creating a computer-aided decision (CAD) framework for the diagnosis of immune system diseases

\section{Abbreviations}

CTD: Connective tissue diseases; ANA: Antinuclear antibody; llf: Indirect immune fluorescence; HEp2: Typically human alveolar epithelial cells type2; FRQI: Flexible representation of the quantum image; MIVIA HEp 2: Data set images; PSNR: Peak signal-to-noise ratio; SA: Segmentation accuracy; DS: Dice similarity; Js: Jaccard similarity; CAD: Computer-aided decision; QEM: Quantum entropy measures; QE: Quantum entropy; MCC: Matthews correlation coefficient

\section{Acknowledgements}

This project was funded by the Deanship of Scientific Research (DSR), King Abdulaziz University, Jeddah, under grant No.(D-111-363-1440). The authors, therefore, gratefully acknowledge the DSR technical and financial support.

\section{Authors' contributions}

The two authors contributed equally to the presented research and writing of this paper. All authors read and approved the final manuscript.

\section{Funding}

This project was funded by the Deanship of Scientific Research (DSR), King Abdulaziz University, Jeddah, Saudi Arabia, under grant No.(D-111-363-1440).

\section{Availability of data and materials}

Availability of data and materials MIVIA HEp-2 image data set. Version: 1.0 For more information and for obtaining the User License Agreement (EULA ). Please refer to the data set web page: https://mivia.unisa.it/datasets/biomedicalimage-datasets/hep2-image-dataset/ [45].

\section{Declarations}

\section{Competing interests}

The authors declare that they have no competing interests.

\section{Author details}

${ }^{1}$ Department of Statistics, Faculty of Science- AL Faisaliah, King Abdulaziz University, 32691, Jeddah, Saudi Arabia.

${ }^{2}$ University of Jeddah, College of Science,Department of Statistics, Jeddah, Saudi Arabia. ${ }^{3}$ Computer College, Suez Canal University, Ismailia, Egypt.

Received: 4 May 2020 Accepted: 14 Dec 2020

Published online: 23 April 2021

\section{References}

1. R. Hiemann, N. Hilger, U. Sack, M. Weigert, Objective quality evaluation of fluorescence images to optimize automatic image acquisition. Cytom. A: J. Int. Soc. Anal. Cytol. 69(3), 182-184 (2006)

2. S. Abdel-Khalek, G. Abdel-Azim, Z. Abo-Eleneen, A.-S. Obada, New approach to image edge detection based on quantum entropy, vol. 37, (2016), pp. 141-154

3. A. D. Brink, Thresholding of digital images using two-dimensional entropies, vol. 25, (1992), pp. 803-808

4. Y. Cai, X. Lu, N. Jiang, A survey on quantum image processing. Chin. J. Electron. 27(4), 718-727 (2018)

5. C.-C. Cheng, T.-Y. Hsieh, J.-S. Taur, Y.-F. Chen, An automatic segmentation and classification framework for anti-nuclear antibody images, vol. 12, (2013), pp. 1-25 
6. S. S. Chouhan, A. Kaul, U. P. Singh, Soft computing approaches for image segmentation: a survey, vol. 77, (2018), pp. 28483-28537

7. C. Creemers, K. Guerti, S. Geerts, K. Van Cotthem, A. Ledda, V. Spruyt, in Proceedings of the 4th International Symposium on Applied Sciences in Biomedical and Communication Technologies, Hep-2 cell pattern segmentation for the support of autoimmune disease diagnosis, (2011), p. 28

8. C.-C. Cheng, T.-Y. Hsieh, J.-S. Taur, Y.-F. Chen, An automatic segmentation and classification framework for anti-nuclear antibody images. Biomed. Eng. Online. 12(1), 1-25 (2013)

9. P. Perner, H. Perner, B. Müller, Mining knowledge for hep-2 cell image classification. Artif. Intell. Med. 26(1-2), 161-173 (2002)

10. U. Sack, S. Knoechner, H. Warschkau, U. Pigla, F. Emmrich, M. Kamprad, Computer-assisted classification of hep-2 immunofluorescence patterns in autoimmune diagnostics. Autoimmun. Rev. 2(5), 298-304 (2003)

11. M. Sezgin, B. Sankur, Survey over image thresholding techniques and quantitative performance evaluation. J. Electron. Imaging. 13(1), 146-166 (2004)

12. K. Doi, Computer-aided diagnosis in medical imaging: historical review, current status and future potential. Comput. Med. Imaging Graph. 31(4-5), 198-211 (2007)

13. A. Rigon, P. Soda, D. Zennaro, G. lannello, A. Afeltra, Indirect immunofluorescence in autoimmune diseases: assessment of digital images for diagnostic purpose. Cytom. B: Clin. Cytom. J. Int. Soc. Anal. Cytol. 72(6), 472-477 (2007)

14. A. Melegari, C. Bonaguri, A. Russo, B. Luisita, T. Trenti, G. Lippi, A comparative study on the reliability of an automated system for the evaluation of cell-based indirect immunofluorescence. Autoimmun. Rev. 11(10), 713-716 (2012)

15. N. Wiebe, A. Kapoor, K. Svore, Quantum algorithms for nearest-neighbor methods for supervised and unsupervised learning. arXiv preprint arXiv:1401.2142 (2014)

16. M. Guţă, W. Kotłowski, Quantum learning: asymptotically optimal classification of qubit states. New J. Phys. 12(12), $123032(2010)$

17. C. A. Trugenberger, Probabilistic quantum memories. Phys. Rev. Lett. 87(6), 067901 (2001)

18. Y. C. Eldar, A. V. Oppenheim, Quantum signal processing. IEEE Signal Proc. Mag. 19(6), 12-32 (2002)

19. M. A. Nielson, I. Chuang, Quantum computation and quantum information. (American Association of Physics Teachers, 2002)

20. S. Du, G. Wu, L. Ma, Y. Ma, et al, Maximum quantum entropy based optimal threshold selecting criterion for thresholding image segmentation. (Bethel, 2014)

21. G. Abdel-Azim, A novel technique for detection of suspicious regions in digital mammogram based on maximum quantum entropy. J. Med. Imaging Health Inform. 6(3), 627-633 (2016)

22. I. Williams, N. Bowring, D. Svoboda, A performance evaluation of statistical tests for edge detection in textured images. Comp. Vision Image Underst. 122, 115-130 (2014)

23. S. Lloyd, M. Mohseni, P. Rebentrost, Quantum algorithms for supervised and unsupervised machine learning. arXiv preprint arXiv:1307.0411 (2013)

24. A. Melegari, C. Bonaguri, A. Russo, B. Luisita, T. Trenti, G. Lippi, A comparative study on the reliability of an automated system for the evaluation of cell-based indirect immunofluorescence, vol. 11, (2012), pp. 713-716

25. A. B. Hamza, Nonextensive information-theoretic measure for image edge detection. J. Electron. Imaging. 15(1), 013011 (2006)

26. C. Yan, B. Gong, Y. Wei, Y. Gao, Deep multi-view enhancement hashing for image retrieval. IEEE Trans. Pattern Anal. Mach Intell. (2020). IEEE

27. R. J. Schalkoff, Digital Image Processing and Computer Vision, vol. 286. (Wiley, New York, 1989)

28. P. Q. Le, A. M. lliyasu, F. Dong, K. Hirota, Efficient color transformations on quantum images. JACIII. 15(6), 698-706 (2011)

29. S. Rödiger, P. Schierack, A. Böhm, J. Nitschke, I. Berger, U. Frömmel, C. Schmidt, M. Ruhland, I. Schimke, D. Roggenbuck, et al, in Molecular Diagnostics, A highly versatile microscope imaging technology platform for the multiplex real-time detection of biomolecules and autoimmune antibodies (Springer, 2012), pp. 35-74

30. C. Yan, B. Shao, H. Zhao, R. Ning, Y. Zhang, F. Xu, 3D room layout estimation from a single RGB image. IEEE Trans. Multimed. 22(11), 3014-3024 (2020)

31. H. Zhao, J. Shi, X. Qi, X. Wang, J. Jia, in Proceedings of the IEEE conference on computer vision and pattern recognition, Pyramid scene parsing network, (Honolulu, 2017), pp. 2881-2890

32. H. Zhao, M. Lu, A. Yao, Y. Guo, Y. Chen, L. Zhang, in Proceedings of the IEEE Conference on Computer Vision and Pattern Recognition, Physics inspired optimization on semantic transfer features: An alternative method for room layout estimation, (Honolulu, 2017), pp. 10-18

33. J. Liu, K. Doi, A. Fenster, S. Chan, in MIPPR 2009: Medical Imaging, Parallel Processing of Images, and Optimization Techniques, Mippr 2009: Medical imaging, parallel processing of images, and optimization techniques, vol. 7497, (Honolulu, 2009)

34. B. Sun, A. M. lliyasu, F. Yan, F. Dong, K. Hirota, An RGB multi-channel representation for images on quantum computers, vol. 17, (2013), pp. 404-417

35. S. Abdel-Khalek, G. Abdel-Azim, Z. Abo-Eleneen, A.-S. Obada, New approach to image edge detection based on quantum entropy. J. Russ. Laser Rese. 37(2), 141-154 (2016)

36. P. Q. Le, F. Dong, K. Hirota, A flexible representation of quantum images for polynomial preparation, image compression, and processing operations. Quantum Inf. Process. 10(1), 63-84 (2011)

37. F. Yan, A. M. lliyasu, S. E. Venegas-Andraca, A survey of quantum image representations. Quantum Inf. Process. 15(1), 1-35 (2016)

38. B. Sun, A. M. Iliyasu, F. Yan, F. Dong, K. Hirota, An RGB multi-channel representation for images on quantum computers. J. Adv. Comput. Intell. Intell. Inform. 17(3), 404-417 (2013)

39. X. Wang, C. Yang, G.-S. Xie, Z. Liu, Image thresholding segmentation on quantum state space. Entropy. 20(10), 728 (2018) 
40. Y. Zhang, K. Lu, Y. Gao, M. Wang, Neqr: a novel enhanced quantum representation of digital images. Quantum Inf. Process. 12(8), 2833-2860 (2013)

41. Y. Cai, X. Lu, N. Jiang, A survey on quantum image processing. Chin. J. Electron. 27(4), 718-727 (2018)

42. P. Sahoo, C. Wilkins, J. Yeager, Threshold selection using renyi's entropy. Pattern Recog. 30(1), 71-84 (1997)

43. A. D. Brink, Thresholding of digital images using two-dimensional entropies. Pattern Recog. 25(8), 803-808 (1992)

44. Z. Yongde, Principles of Quantum Information Physics [M]. (Science Press, Beijing, 2005)

45. P. Foggia, G. Percannella, P. Soda, M. Vento, Benchmarking hep-2 cells classification methods. IEEE Trans. Med. Imaging. 32(10), 1878-1889 (2013)

46. S. S. Chouhan, A. Kaul, U. P. Singh, Soft computing approaches for image segmentation: a survey. Multimed. Tools Appl. 77(21), 28483-28537 (2018)

\section{Publisher's Note}

Springer Nature remains neutral with regard to jurisdictional claims in published maps and institutional affiliations.

\section{Submit your manuscript to a SpringerOpen ${ }^{\circ}$} journal and benefit from:

- Convenient online submission

- Rigorous peer review

- Open access: articles freely available online

- High visibility within the field

- Retaining the copyright to your article

Submit your next manuscript at $\boldsymbol{\triangleright}$ springeropen.com 